\title{
Joint User Clustering and Power Allocation for Vehicular Communications
}

\author{
Qian CHEN, Gongbin QIAN ${ }^{1}$, Chunlong HE, Rujun ZHAO and Yuping ZHENG \\ College of Information Engineering, Shenzhen University, Shenzhen, 518060, China
}

\begin{abstract}
Device-to-device (D2D) communication has emerged as a promising concept for supporting the vehicular networks, which can efficient and reliable enhance cellular network. In this paper, we discuss two different design criteria for vehicular networks. They are maximizing overall vehicle-to-infrastructure (V2I) link throughput while guaranteeing the minimum reliability for each vehicle-tovehicle (V2V) link, and maximizing the minimum throughput of all V2V links under the constraints of minimum V2I link throughput requirements. Because both of these problems is an mixed integer non-linear programming problem, we solve these problems in two steps, i.e., by first clustering D2D users into clusters and then optimizing their respective power allocations. Specifically, we first propose a spectral clustering (SC) method for D2D users clustering. Then, two power allocation algorithms are developed to maximize the sum V2I link throughput and maximize the minimum V2V link throughput, respectively. The effectiveness of proposed resource allocation algorithms is validated by computer simulation.
\end{abstract}

Keywords. Vehicular communications, D2D communication, resource allocation

\section{Introduction}

In recent years, the vehicular networks have attracted more and more attention with the rapid development of the Intelligent Transport System (ITS) [1] [2]. As a potential application scenario of the fifth generation of mobile communication systems $(5 \mathrm{G})$, vehicular communication networks require large bandwidth, low latency and high reliability [3].

Vehicle communication networks is considered to support three applications, namely road safety, traffic efficiency and infotainment. Infotainment and traffic efficiency applications usually are based on vehicle-to-infrastructure (V2I) communications with large bandwidth requirement. Meanwhile, the road safety application is based on vehicle-tovehicle (V2V) communications with high reliability and low delay [4] [5]. To achieve this goal, the device-to-device (D2D) communication assisted cellular networks is considering to be a feasible solution for vehicular communication networks.

Resource allocation for vehicular communication networks have been extensively researched. In [6], an efficient resource allocation scheme is studied for D2D-enabled vehicular networks. Indeed, the presented algorithms results in improved system performance, however, each resource block is used by only one CUE and DUE. Similarly, a

\footnotetext{
${ }^{1}$ Corresponding Author: College of Information Engineering, Shenzhen University, Shenzhen, 518060, China; E-mail: qiangb@szu.edu.cn.
} 
robust resource allocation algorithm has been proposed in [7] [8] to improve the system performance of vehicular network. By exploit graph partitioning algorithm, the authors in [9] has proposed an efficient resource allocation scheme, when considering the multiple DUE share the same resource block (RB) with one CUE. Further, in [10], a reinforcement learning algorithm for the D2D-based vehicular networks has been developed.

In this paper, we will investigate two different design criteria for vehicular services with different needs, including maximizing the overall V2I links throughput and maximizing the minimum throughput of all V2V links. Firstly, we adjust those V2V links that cause more harmful interference to other disjoint spectrum sharing clusters by exploit spectral clustering (SC) algorithm. Then, in one scenario of infotainment applications (e.g., movie/video sharing), we will consider maximizing overall V2I link throughput while guaranteeing the minimum reliability for each V2V link. For this criterion, we can derive a power allocation algorithm to maximize the capacity of each cluster by utilizing the monotonicity of the objective function as same as [9]. In other scenario of security applications, we will consider maximizing the minimum throughput of all V2V links under the constraints of minimum V2I link throughput requirements. For this criterion, we will introducing an auxiliary variable and a sequential convex approximation based iterative method to perform power allocation.

The rest of this paper is organized as follows. In Section 2, the vehicular communication networks model is given. In Section 3, we get V2V clustering result by using a simple algorithm. Section 4 and 5 investigate the power allocation problems with different optimization problem. Section 6 , the numerical results and discussion are provided. We conclusion the paper in Section 7.

\section{System Model}

This paper considers a D2D-based vehicular communication networks, as shown in Fig.1. The considered networks consist of $M$ V2I high-capacity communication for some infotainment services and $K \mathrm{~V} 2 \mathrm{~V}$ high-reliability communication by using D2D communications fashion for some security services, and $K \gg M$. For notational simplicity, we denote the set of V2I links and V2V links by $\mathcal{M}$ and $\mathcal{K}$ respectively, i.e., $\mathcal{M} \triangleq\{1,2, \ldots, M\}$ and $\mathcal{K} \triangleq\{1,2, \ldots, K\}$. We consider the case that each V2I channel is reused by multiple V2V users, and V2I links using orthogonal frequency division multiple access (OFDMA) technology to access the cellular networks. Without loss of generality, we assume that both the BS and vehicular are equipped with single antenna and the perfect channel state information(CSI) is available at both the BS and vehicular. The signal to interference plus noise ratio (SINR) of the $m$ th V2I link can be expressed

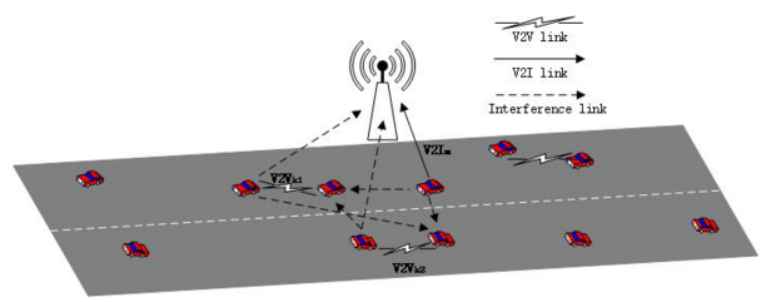

Figure 1. The considered D2D-based vehicular communication model 


$$
\gamma_{m}^{c}=\frac{P_{m}^{c} g_{m, B}}{\sigma^{2}+\sum_{k=1}^{K} \rho_{m, k} P_{k}^{d} g_{k, B}}
$$

where $P_{m}^{c}$ and $P_{k}^{d}$ denotes the transmit power of the $m$ th V2I transmitter and the $k$ th $\mathrm{V} 2 \mathrm{~V}$ transmitter, respectively. $g_{m, B}$ is the channel gain between the $k$ th $\mathrm{V} 2 \mathrm{~V}$ transmitter and the BS . $\rho_{m, k}$ is a binary parameter, $\rho_{m, k}=1$, means that the $k$ th $\mathrm{V} 2 \mathrm{~V}$ pair reuses the $k$ th V2I's channel; otherwise, $\rho_{m, k}=0 . \sigma^{2}$ represents the power of complex additive white Guassian noise(AWGN) of both V2I links and V2V links. Similarly, the SINR of the $k$ th $\mathrm{V} 2 \mathrm{~V}$ link can be written as

$$
\gamma_{k}^{d}=\frac{P_{k}^{d} g_{k}}{\sigma^{2}+\sum_{m=1}^{M} \rho_{m, k} P_{m}^{k} g_{m, k}+\sum_{k^{\prime}=1, k^{\prime} \neq k}^{K} \rho_{k^{\prime}, k} P_{k^{\prime}}^{d} g_{k^{\prime}, k}},
$$

where $g_{k}$ is the channel gain between the $k$ th $\mathrm{V} 2 \mathrm{~V}$ transmitter and the $k$ th $\mathrm{V} 2 \mathrm{~V}$ receiver. $g_{m, k}$ is the channel gain between the $k$ th V2I transmitter and the $k$ th V2V receiver. $g_{k^{\prime}, k}$ is the channel gain between the $k^{\prime}$ th $\mathrm{V} 2 \mathrm{~V}$ transmitter and the $k$ th $\mathrm{V} 2 \mathrm{~V}$ receiver.

\section{V2V Clustering}

In order to solve the strong interference that is caused by multiple V2V links sharing the same V2I links channel, we need to design appropriate channel reuse strategies to assign all V2V links to different clusters. Based on minimizing the mutual interference criterion, we can design the following V2V clustering scheme.

First, we transform the $\mathrm{V} 2 \mathrm{~V}$ clustering problem into a graph partition problem, where each vehicle and channel are interpreted as the vertices and weighted edges of the graph, specifically, the problem is a NP-hard problem. Obviously, in a graph, all the sum of the edge weights is a fixed value, the cut target can be regard as the weight in all clusters that minimizes the sum, i.e., maximizing the sum of the weights between the clusters. Next, we will use spectral clustering (SC) algorithm to solve this problem.

Spectral clustering is an algorithm evolved from graph theory with small computational complexity and simple implementation. A simple spectral clustering algorithm has been used in [11]. For the proposed problem, we try to make all the reciprocal of the channel gain of all channels as the edges of graph. The goal of our algorithm is to assign $K$ users to $n$ clusters, $C_{1}, \cdots, C_{n}$, in this paper, $n=M$. The V2V clustering algorithm is shown in the Table 1.

Specifically, $W=\left[1 / w_{k^{\prime}, k}\right]$ denotes the similarity matrix of the proposed graph, $w_{k^{\prime}, k}$ is the weight of the graph's edge, which is define the reciprocal of channel power gain $g_{k, k^{\prime}} . \mathrm{D}$ is denoted as a diagonal degree matrix with $d_{k, k}=\sum_{k^{\prime}=1}^{K} w_{k, k^{\prime}}$. 
Table 1. V2V Clustering Algorithm

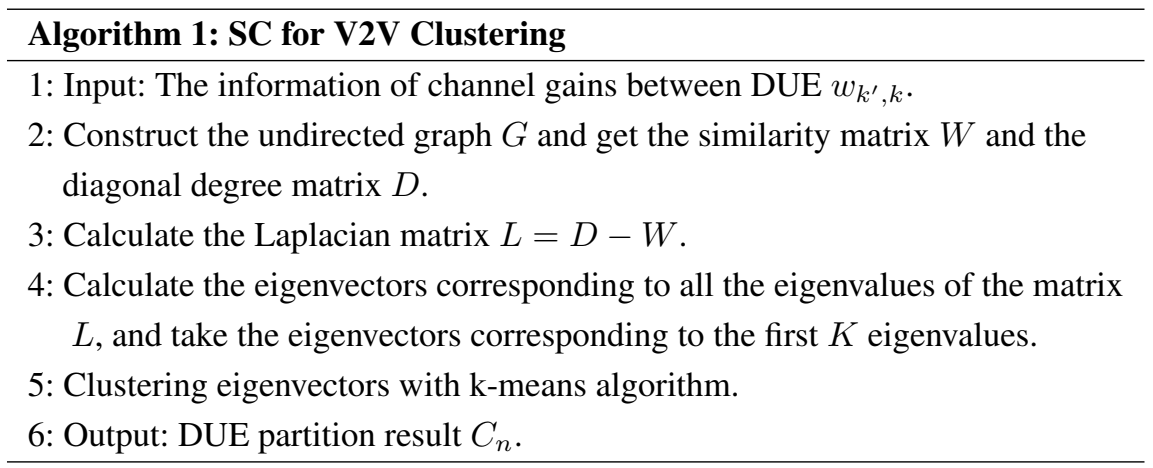

\section{Maximum Sum of V2I Links Capacity Optimization}

In this section, we consider maximizing the sum capacity of V2I links under the constraints of satisfying the requirements of the minimum V2I transmit rate, maximum transmit power of each vehicle, and guaranteeing the minimum reliability for each V2V links. In addition, we transfer the reliability of each $\mathrm{V} 2 \mathrm{~V}$ links to the probability of outage events, i.e. V2V received SINR is below a predetermined threshold $\gamma_{0}^{d}$. The problem can be written as

$$
\begin{array}{ll}
\max _{\mathbf{P}_{m}^{c}, \mathbf{P}_{k}^{d}} & \sum_{m=1}^{M} \log _{2}\left(1+\frac{P_{m}^{c} g_{m, B}}{\sigma^{2}+\sum_{k \in C_{m}} P_{k}^{d} g_{k, B}}\right) \\
\text { s.t. } & 0 \leq P_{m}^{c} \leq P_{\max }^{c}, \\
& 0 \leq P_{k}^{d} \leq P_{\max }^{d}, \\
& P_{r}\left\{\frac{P_{k}^{d} g_{k}}{\sigma^{2}+P_{m}^{c} g_{m, k}+\sum_{k^{\prime}=1, k^{\prime} \neq k}^{K} P_{k^{\prime}}^{d} g_{k^{\prime}, k}} \leq \gamma_{0}^{d}\right\} \leq p_{0} .
\end{array}
$$

In the problem (3), constraint (3a) and (3b) represent V2I link's and V2V link's maximum power limit, where $P_{\max }^{c}$ and $P_{\max }^{d}$ are the maximum transmit power of each V2I transmitter and V2V transmitter, respectively. Constraint $(3 \mathrm{c})$ represent the reliability requirements for each $\mathrm{V} 2 \mathrm{~V}$ link, $R_{\min }^{c}$ and $\gamma_{0}^{d}$ denote the minimum capacity needed by V2I link and the minimum SINR requirement of V2V link. $P_{r}\{\cdot\}$ evaluates the probability of the input and $p_{0}$ is the tolerable outage probability for V2V links.

From [9], the constraint (3d) can be transformed into definite boundaries. From the previous discussion, we have get a $\mathrm{V} 2 \mathrm{~V}$ clustering result, next, we want to find the maximum capacity of all possible sharing patterns of V2I and V2V clustering. So the problem can be transformed as 


$$
\max _{\mathbf{P}_{m}^{c}, \mathbf{P}_{k}^{d}} \sum_{m=1}^{M} \log _{2}\left(1+\frac{P_{m}^{c} g_{m, B}}{\sigma^{2}+\sum_{k \in C_{m}} P_{k}^{d} g_{k, B}}\right)
$$

s.t. The constraints in 3(a), 3(b),

$$
\frac{P_{k}^{d} g_{k}}{\sigma^{2}+P_{m}^{c} g_{m, k}+\sum_{k^{\prime}=1, k^{\prime} \neq k}^{K} P_{k^{\prime}}^{d} g_{k^{\prime}, k}} \geqslant \frac{\gamma_{0}^{d}}{-\ln \left(1-p_{0}\right)} .
$$

Notice that the objective function (4) is monotonically nonincreasing in terms of $P_{k}^{d}$, from the (4b), the optimal $P_{k}^{d}$ in the $n$th cluster must be achieved at

$$
\widetilde{P}_{k}^{d}=\frac{\widetilde{\gamma}_{0}}{g_{k}}\left(\sigma^{2}+P_{m}^{c} g_{m, k}+\sum_{k^{\prime}=1, k^{\prime} \neq k}^{K} P_{k^{\prime}}^{d} g_{k^{\prime}, k}\right),
$$

where $\widetilde{\gamma}_{0}=-\frac{\gamma_{0}^{d}}{\ln \left(1-p_{0}\right)}$. By substituting $\widetilde{P}_{k}^{d}$ into (5), we can obtain the optimal $\widetilde{P}_{m}^{c}$ as

$$
\widetilde{P}_{m}^{c}=\left\{P_{\max }^{c},\left\{\frac{P_{\max }^{d} g_{k}-\left(\sigma^{2}+\sum_{k^{\prime}=1, k^{\prime} \neq k}^{K} P_{\max }^{d} g_{k^{\prime}, k}\right) \widetilde{\gamma}_{0}}{g_{m, k} \widetilde{\gamma}_{0}}\right\}\right\}
$$

Next, we can find the best spectrum multiplexing partner between the V2I link and V2V link cluster sets using the Hungarian method [6] by constructing a bipartite graph.

The developed power allocation algorithm is showed in Table 2. For convenience, sum of V2I links capacity can be written as $R_{m, n}^{c}$.

Table 2. Maximum sum V2I link capacity algorithm

\begin{tabular}{l} 
Algorithm 2: Maximum sum V2I link capacity \\
\hline 1:for $\mathbf{m}=\mathbf{1 : M}$ do \\
2: for $\mathbf{n = 1 : M ~ d o ~}$ \\
3: Obtain the optimal power allocation $\left(P_{m}^{c}, P_{k}^{d}\right)$ from \\
$\quad(6)$ and (7) for the single CUE-DUE cluster. \\
4: Calculate $R_{m}^{c}$ according to equations (4). \\
5: end for \\
6:end for \\
7:Use the Hungarian method to find the optimal reuse \\
pattern $\rho_{m}^{*}$ based on $R_{m, n}^{c}$. \\
8:Return the optimal spectrum reuse pattern $\rho_{m, k}^{*}$ and \\
the corresponding power allocation $\left(P_{m}^{c}, P_{k}^{d}\right)$.
\end{tabular}




\section{Maximum the Minimum V2V Link Capacity Optimization}

The maximizing the sum V2V link capacity problem in section IV aims to ensure V2I link's large capacity services. However, in many security applications, a balance connectivity for V2V links rather than a maximized sum V2V capacity, is more desirable. In this section, we mainly study maximizing the minumum achievable rate among all the V2V links while guaranteeing the minimum V2I link throughput requirements. The problem can be formulated as

$$
\max _{\mathbf{P}_{m}^{c}, \mathbf{P}_{k}^{d}} \min _{k \in \mathcal{K}} \log _{2}\left(1+\frac{P_{k}^{d} g_{k}}{\sigma^{2}+P_{m}^{c} g_{m, k}+\sum_{k^{\prime}=1, k^{\prime} \neq k}^{K} P_{k^{\prime}}^{d} g_{k^{\prime}, k}}\right)
$$

s.t. The constraints in (3a), 3(b).

$$
\log _{2}\left(1+\frac{P_{m}^{c} g_{m, B}}{\sigma^{2}+\sum_{k \in C_{m}} P_{k}^{d} g_{k, B}}\right) \geq R_{\min }^{c}
$$

Constraint (7b) represents the minimum throughput requirement for V2I link. In general, problem (7) is a generalized fractional linear programming problem, which can be solved by parametric linear programming [12]. By introduce an auxiliary variable $\tau$ and add a constraint that all V2V links not less than $\tau$, we can transform (7) into the standard form of convex optimization problem with inequality constraints as follow

$$
\max _{\mathbf{P}_{m}^{c}, \mathbf{P}_{k}^{d}} \tau
$$

s.t. The constraints in (3a), 3(b), 7(b),

$$
\log _{2}\left(1+\frac{P_{k}^{d} g_{k}}{\sigma^{2}+P_{m}^{c} g_{m, k}+\sum_{k^{\prime}=1, k^{\prime} \neq k}^{K} P_{k^{\prime}}^{d} g_{k^{\prime}, k}}\right) \geq \tau .
$$

According to [7], the original problem (7) can be solved through solving the equivalent problem (8) by using a sequential convex approximation based iterative algorithm. Then, we can using the Hungarian method to find optimal spectrum reuse scheme.

\section{Simulation Results}

In this section, we compare different resource allocation criteria through simulation results. We follow the simulation setup for the freeway case in [9], and assume that $K=10$ and $M=30$. Firstly, we compare the performance of our proposed SC algorithm with 


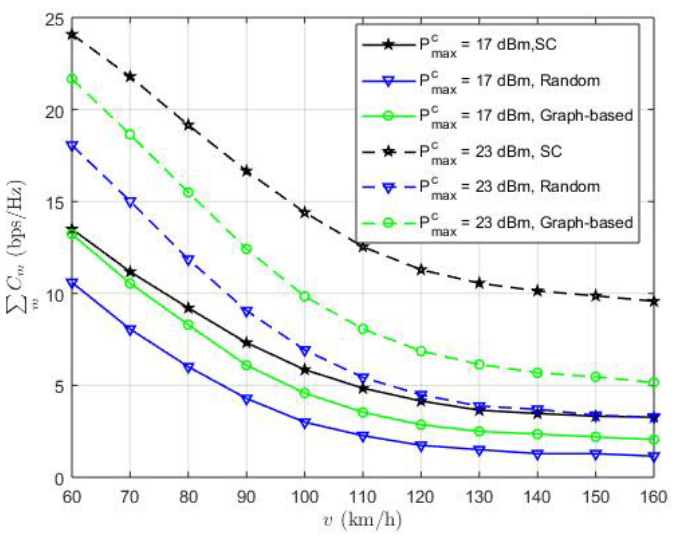

Figure 2. Sum capacity of V2I links.

the Graph-Based method in [13] and a random partitioning algorithm. And then we compare the three different power allocation methods.

Fig.2 compares the sum of V2I link throughput versus the vehicle speed when using different clustering algorithms. From Fig.2, we can obtain that the sum of V2I link throughput is decreased with the growth of vehicles speed. This is because higher speed will make the power limit of V2I link more stringent and reduce the V2I link transmission power. Fig. 2 also shows the impact of different V2V clustering methods. It can be seen from Fig.2, compared with Graph-Based and random partitioning methods, the $\mathrm{SC}$ method has a better performance. The reason is that the SC method can reduce the intra-cluster interference.

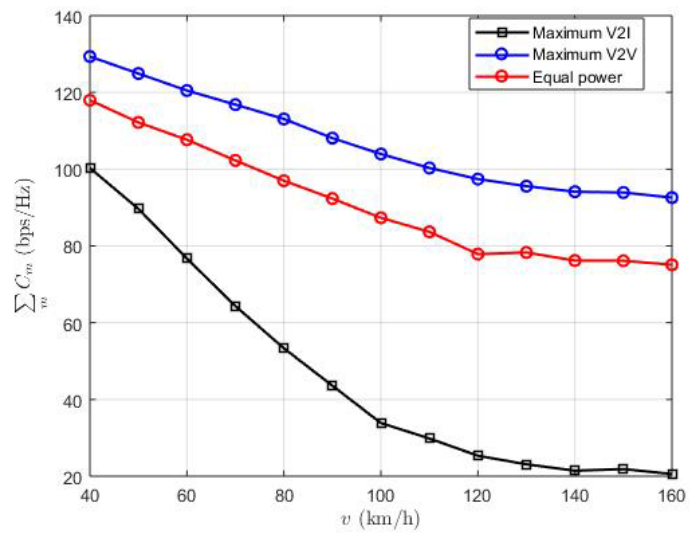

Figure 3. Minimum capacity of V2V links.

Fig. 3 compares the minimum throughput of V2V link among maximizing the V2V link minimum throughput scheme, maximum sum V2I link throughput scheme and equal power allocation scheme. Compared with maximum sum V2I link throughput and equal power allocation, it is observed in Fig. 3 that the proposed scheme outperforms the other 
two allocation schemes in terms of minimum throughput. This illustrates the necessity of choosing the right power allocation method for different vehicular applications. Specifically, we can using maximum sum V2I link throughput scheme in infotainment and traffic efficiency applications, and using maximizing the V2V link minimum throughput scheme in safety applications.

\section{Conclusion}

In this paper, we have compared two different design criteria for vehicular networks. We first exploiting the SC method to take D2D user into different cluster, and then two optimal power allocation schemes for maximizing the overall V2I link throughput while guaranteeing the minimum reliability for each V2V link, and maximizing the minimum throughput of all V2V links under the constraints of minimum V2I link throughput requirements, respectively. Simulation results showed effectiveness of proposed schemes.

However, because of the computational complexity of solving power allocation problem, the proposed resource allocation is difficult to implement. Future works will focus on developing low complexity resource allocation algorithms, such as machine learning method.

\section{Acknowledgement}

This work was supported in part by Shenzhen Overseas High-level Talents Innovation and Entrepreneurship under Grant KQJSCX20180328093835762, in part by the Shenzhen Basic Research Program under Grants JCYJ20190808122409660 and JCYJ20170412104656685.

\section{References}

[1] G. S. Khekare and A. V. Sakhare, "A smart city framework for intelligent traffic system using vanet," in International Multi-conference on Automation, 2013, pp. 302-305.

[2] G. S. Khekare, "Design of emergency system for intelligent traffic system using vanet," in International Conference on Information Communication \& Embedded Systems, 2014.

[3] G. Araniti, C. Campolo, M. Condoluci, A. Iera, and A. Molinaro, "LTE for vehicular networking: a survey," IEEE Communications Magazine, vol. 51, no. 5, pp. 148-157, May 2013.

[4] L. Liang, J. Kim, S. C. Jha, K. Sivanesan, and G. Y. Li, "Spectrum and power allocation for vehicular communications with delayed CSI feedback," IEEE Wireless Communications Letters, vol. 6, no. 4, pp. 458-461, Aug. 2017.

[5] L. Liang, H. Peng, G. Y. Li, and X. Shen, "Vehicular communications: A physical layer perspective," IEEE Transactions on Vehicular Technology, vol. 66, no. 12, pp. 10647-10659, Dec. 2017.

[6] L. Liang, G. Y. Li, and W. Xu, "Resource allocation for D2D-Enabled vehicular communications," IEEE Transactions on Communications, vol. 65, no. 7, pp. 3186-3197, Apr. 2017.

[7] K. Doppler, M. Rinne, C. Wijting, C. B. Ribeiro, and K. Hugl, "Device-to-device communication as an underlay to LTE-advanced networks," IEEE Communications Magazine, vol. 47, no. 12, pp. 42-49, Dec. 2009.

[8] S. Bharati and P. Podder, "Adaptive PAPR reduction scheme for OFDM using SLM with the fusion of proposed clipping and filtering technique in order to diminish PAPR and signal distortion," Wireless Personal Communications, no. 1, pp. 1-18, 2020. 
[9] L. Liang, S. Xie, G. Y. Li, Z. Ding, and X. Yu, "Graph-based resource sharing in vehicular communication,” IEEE Transactions on Wireless Communications, vol. 17, no. 7, pp. 4579-4592, Apr. 2018.

[10] G. Khekare, P. Verma, U. Dhanre, S. Raut, and S. Sheikh, "The optimal path finding algorithm based on reinforcement learning," The optimal path finding algorithm based on reinforcement learning, vol. 12, 2020.

[11] B. Golkar and E. Sousa, "Adaptive partitioned interference management in cellular networks," in in Proc. 2012 IEEE GLOBECOM. Anaheim, CA, USA: IEEE, Dec. 2012, pp. 4261-4266.

[12] Z. Luo and S. Zhang, "Dynamic spectrum management: Complexity and duality," IEEE Journal of Selected Topics in Signal Processing, vol. 2, no. 1, pp. 57-73, Feb. 2008.

[13] R. Y. Chang, Z. Tao, J. Zhang, and C. . J. Kuo, "Multicell ofdma downlink resource allocation using a graphic framework," IEEE Transactions on Vehicular Technology, vol. 58, no. 7, pp. 3494-3507, 2009. 